\title{
How can we assess the effects of urban environment on obesity using aggregated data?
}

\author{
Seon-Ju Yi ${ }^{1}$ and Changwoo Shon ${ }^{\star 2}$ \\ ${ }^{1}$ Seoul National University, Seoul, Korea (the Republic of); ${ }^{2}$ Seoul Institute, Seoul, Korea (the Democratic People's Republic of)
}

\section{Objective}

This study aimed to assess the effects of urban physical environment on individual obesity using geographically aggregated health behavior surveillance data applying a geo-imputation method.

\section{Introduction}

'Where we live' affects 'How we live'. Information about 'how one lives' collected from the public health surveillance data such as the Behavioral Risk Factor Surveillance System (BRFSS). Neighborhood environment surrounding individuals affects their health behavior or health status are influenced as well as their own traits. Meanwhile, geographical information of subjects recruited in the health behavior surveillance data is usually aggregated at administrative levels such as a county. Even if we do not know accurate addresses of individuals, we can allocate them to the random locations where is analogous to their real home within a locality using a geo-imputation method. In this study, we assess the association between obesity and built environment by applying random property allocation (1).

\section{Methods}

Data from the Korean Community Health Survey (KCHS), which is the nationwide community-based cross-sectional survey conducted by 253 community health centers in South Korea, were used (2). More than 90000 subjects recruited in the capital city Seoul from 2011 to 2014. They were selected by two-step stratified random sampling (424 administrative communities with an average area of $1.16 \mathrm{~km}^{2}$ and two house types) in each 25 counties. We re-allocated them randomly on the nested locality based on their community (administrative boundaries) and hose type (land-use) using GIS program (Figure 1). Surrounding built environment elements such as fast-food markets, driving roads, public transit and road-crosse were measured within $500 \mathrm{~m}$ buffer from randomly allocated locations as density or distance. Variables associating obesity are measured by : 1) self-reported obesity (self-reported body mass index(BMI) $\geq 25$ ) (Figure 2), 2) perceived obesity, 3) intention to weight control. We implemented logistic regression models to estimate the effect of physical environmental factors on obesity.

\section{Results}

The person who lives in a detached house, nearer fast food markets or with higher driving road density was more likely to be obese. Those who live in a detached house was less perceived their obesity. Those who live in a detached house, nearer fast food markets or with higher driving road density was less likely to intend to control their body weights. An association between intention to weight control and accessibility to subway station showed marginal effect.

\section{Conclusions}

Urban environments influenced individual's obesity, perception, and intention to weight loss. Since we used cross-sectional survey data, we do not account cumulative environmental influence. Moreover, individuals' self-selection of more healthier places were not accounted. Even though we did not measure the environment at individuals' real address, we can measure the effects of neighborhood environment more efficiently by using random property allocation.

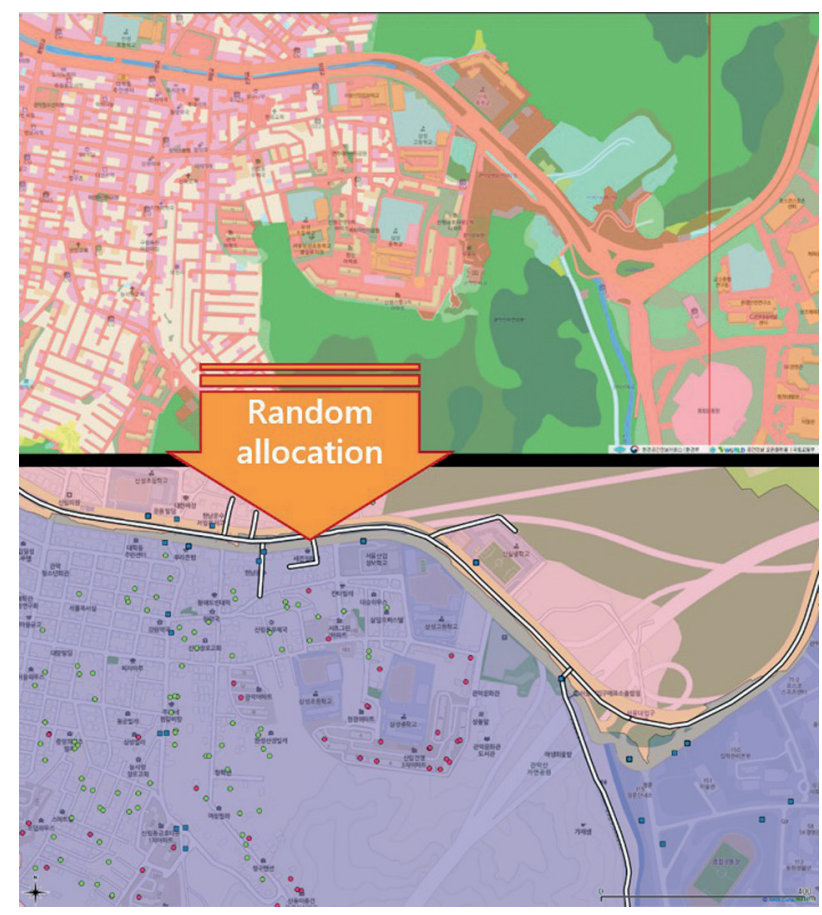

Figure 1. Land use map (up) and result of random allocation (down) in Daehakcong, Gwanakgu, Seoul.png

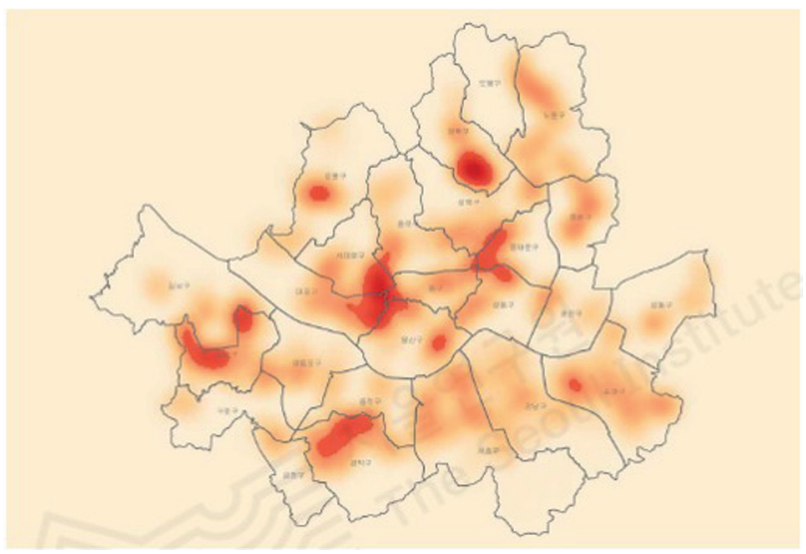

Distribution of obese population in Seoul, South Korea

\section{Keywords}

random property allocation; BRFSS; obesity; built environment; South Korea

\section{Acknowledgments}

This study was supported by the Seoul Institute and the Seoul Metropolitan Government. 


\section{References}

1. Walter SR, Rose N. Random property allocation: A novel geographic imputation procedure based on a complete geocoded address file. Spatial and spatio-temporal epidemiology. 2013;6:7-16. Epub 2013/08/27. doi: 10.1016/j.sste.2013.04.005. PubMed PMID: 23973177

2. Kim YT, Choi BY, Lee KO, Kim H, Chun JH, Kim SY, et al. Overview of Korean Community Health Survey. J Korean Med Assoc. 2012;55(1):74-83. (in Korean)

\section{*Changwoo Shon}

E-mail: cwshon21@si.re.kr 